NOTE

\title{
Corresponding dose response of radiographic film with layered Gafchromic film
}

\author{
Martin J Butson ${ }^{1,2}$, Tsang Cheung ${ }^{1}$ and Peter K N Yu ${ }^{1}$ \\ ${ }^{1}$ Department of Physics and Materials Science, City University of Hong Kong, Kowloon Tong, \\ Hong Kong \\ 2 Illawarra Cancer Care Centre, Department of Medical Physics, Crown Street, Wollongong, \\ NSW 2500, Australia \\ E-mail: butsonm@iahs.nsw.gov.au
}

Received 15 August 2002

Published 30 October 2002

Online at stacks.iop.org/PMB/47/N285

\begin{abstract}
This note investigates the dose response of layered HS Gafchromic film compared to Kodak EDR-2 radiographic film. Using five layers of HS type Gafchromic film a dose response greater than EDR-2 film is achieved at the peak wavelength $(0.55 \mathrm{OD} / \mathrm{Gy}$ versus $0.3 \mathrm{OD} / \mathrm{Gy}$ for EDR-2 film). Even over a broader waveband of $30 \mathrm{~nm}$, which is similar to that found in ultra bright LED scanners, the response was found to be $0.38 \mathrm{OD} / \mathrm{Gy}$ as opposed to $0.29 \mathrm{OD} / \mathrm{Gy}$. Measurements averaged over the entire visible spectrum produce a relative dose response of $0.165 \mathrm{OD} / \mathrm{Gy}$ for five layer HS and $0.29 \mathrm{OD} / \mathrm{Gy}$ for EDR-2 film. Due to this high dose response that is achievable, the five layer HS could be used in applications where small doses are delivered to certain areas and a low dependence of energy response is required for measurement.
\end{abstract}

\section{Introduction}

Radiochromic film, due to its relatively low energy dependence compared to radiographic film, has become a significant dosimetry tool in high-energy radiotherapy (Meigooni et al 1996, Zhu et al 1997). With the introduction of the relatively new HS Gafchromic film the dose sensitivity has been increased by approximately a factor of 2 compared to MD-55-2 film (Butson et al 2002). This is still however a relatively low dose response compared to radiographic film such as Kodak ERD-2 film, which is used in applications such as intensity modulated radiotherapy dose verification. One limitation of radiographic film is its energy response, whereby a large over response is recorded at low x-ray energies. With the use of Gafchromic film HS layers a high dose response detector, which is relatively energy independent, can be created (Butson et al 2002). This short note investigates the optical density properties of a layered HS film detector and compares results to EDR-2 radiographic film in the visible region. 


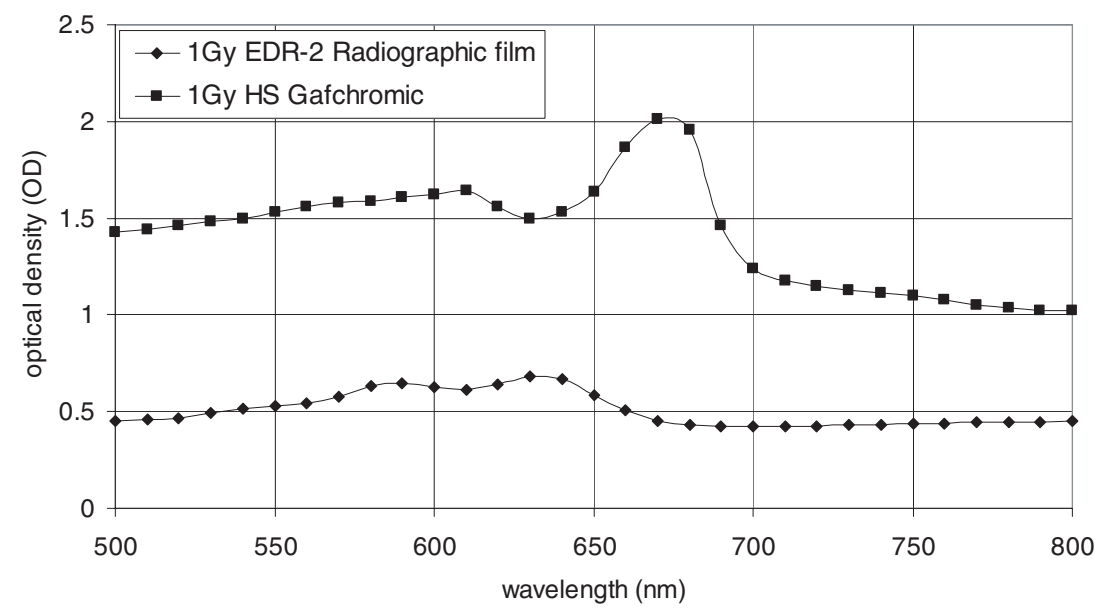

Figure 1. The gross optical density results over the spectral range of $500 \mathrm{~nm}$ to $800 \mathrm{~nm}$ for the five layers of HS Gafchromic film and one layer of EDR-2 radiographic film are shown.

\section{Materials and methods}

Absorbed dose delivery was performed using a Varian 2100C accelerator at a photon energy of $6 \mathrm{MV}$ in an RMI $30 \times 30 \mathrm{~cm}^{2}$ solid water (Constantinou et al 1982) slab phantom at $100 \mathrm{~cm}$ source to surface distance at the $D_{\max }$ position. The films used were Gafchromic HS with batch number I0144HS and Kodak EDR-2 radiographic film. The Gafchromic and radiographic film absorption spectra were measured using a Shimadzu UV-160 UVvisible recording spectrophotometer. Its wavelength range is $200-1100 \mathrm{~nm}$ and has a spectral bandwidth of $3 \mathrm{~nm}$ with an accuracy of $+0.5 \mathrm{~nm}$. The film was held in quartz holding containers during analysis. Measurements were made in $5 \mathrm{~nm}$ intervals from $400 \mathrm{~nm}$ to $800 \mathrm{~nm}$. The radiographic film was processed in a Kodak M35 X-Omat. The high sensitivity HS film dosimeter was constructed using five strips of HS film (dimensions $2 \mathrm{~cm} \times 2 \mathrm{~cm}$ ) films placed in a stack and stuck together with unidirectional bonding tape. The tape only covered the outer $1 \mathrm{~mm}$ edges of each dosimeter. Precautions in handling of radiochromic film outlined in TG-55 (Niroomand-Rad et al 1998) were used. The films were irradiated in 50 cGy intervals up to $300 \mathrm{cGy}$ and spectral analysis performed. The experiments and film analysis were performed at temperatures of $22{ }^{\circ} \mathrm{C} \pm 2{ }^{\circ} \mathrm{C}$ and readout after a $24 \mathrm{~h}$ period from irradiation thus reducing the effects of time- and temperature-dependent evolution (McLaughlin et al 1991, Reinstein et al 1997) of Gafchromic film and the temperature-dependent absorption spectra of the film. The Gafchromic film is only removed from a light-tight envelope during irradiation and readout to reduce the effects of ambient light (Butson et al 1998).

\section{Results and discussion}

Figure 1 shows the gross optical density over the spectral wavelengths of $500-800 \mathrm{~nm}$ for EDR-2 radiographic film and five layers of HS Gafchromic film which were both irradiated to 1 Gy dose. The gross optical density results for the five layers of HS are higher than EDR-2 for most wavelengths with the absorption peak located at approximately $675 \mathrm{~nm}$. For EDR-2 film the gross optical density is lower for a 1 Gy irradiation with smaller peaks located at approximately $580 \mathrm{~nm}$ and $630 \mathrm{~nm}$. Net optical density results, which are shown in future 


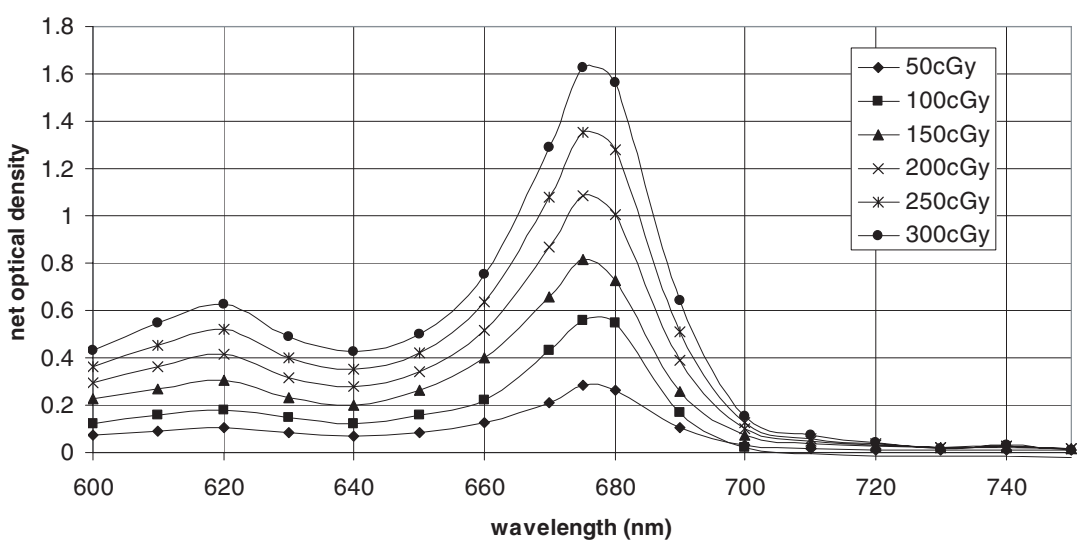

(a)

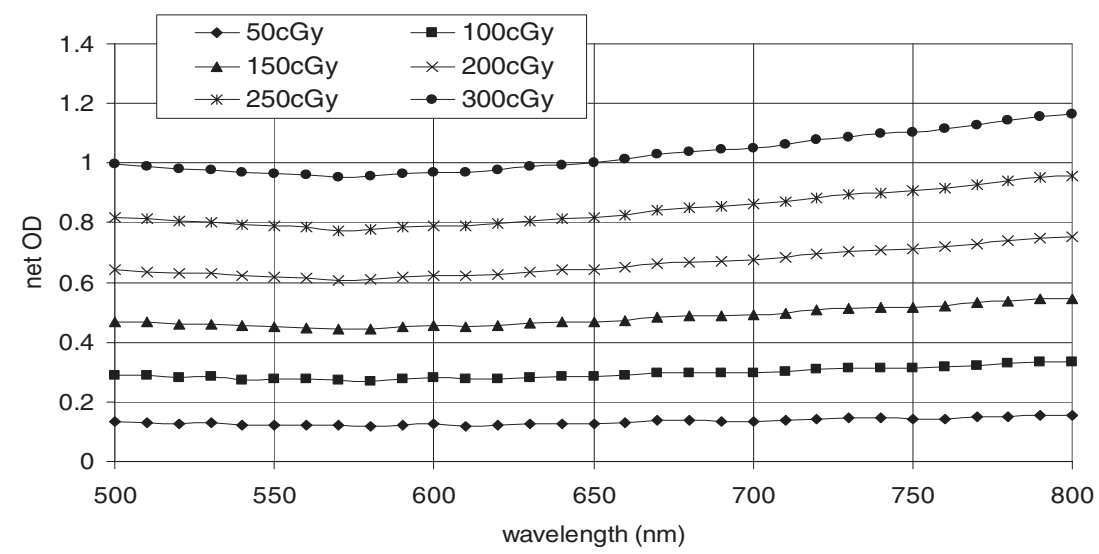

(b)

Figure 2. Parts (a) and (b) show the net optical density change as a function of wavelength for doses ranging from $50 \mathrm{cGy}$ up to $300 \mathrm{cGy}$ for five layers of HS and one layer of EDR-2, respectively.

figures, are the subtraction of the absolute gross density for 0 Gy irradiations from the given dose level films shown.

Figure 2(a) shows the net optical density results for wavelengths of analysis from $600 \mathrm{~nm}$ to $750 \mathrm{~nm}$ for five layers of HS Gafchromic film. The two main absorption peaks occur within this region. A relatively linear increase in OD occurs at most wavelengths with the maximum sensitivity occurring at $675 \mathrm{~nm}$ with a sensitivity of $0.55 \mathrm{OD} / \mathrm{Gy}$ (optical density change per unit dose delivered). Figure 2(b) shows similar results but for EDR-2 radiographic film in the wavelength range of 500-800 $\mathrm{nm}$. A relative minimum in sensitivity occurs at approximately $570 \mathrm{~nm}$ for this film with the maximum sensitivity occurring at greater wavelengths in the infrared region. This variation in response for EDR-2 film is approximately $20 \%$ over the wavelengths tested. However at $675 \mathrm{~nm}$ (peak for HS Gafchromic film) an approximate 0.32 net ODGy change is seen for this film. Figure 3 highlights the variations in optical density response for the EDR-2 radiographic film and HS Gafchromic film in the wavelength range of 600-750 nm. Results show net OD for doses of $100 \mathrm{cGy}$ and $200 \mathrm{cGy}$. As can be seen, the five layers of HS film produce a larger response to dose within the wavelength range of approximately $665-685 \mathrm{~nm}$ which corresponds to the major peak. As some Gafchromic 


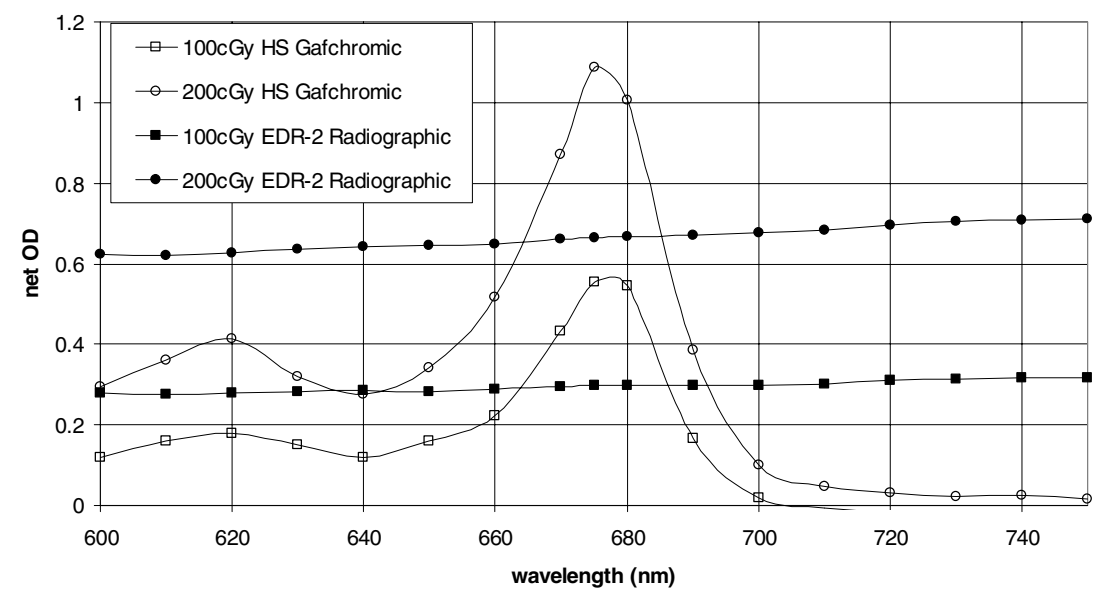

Figure 3. A comparative dose response of the HS and EDR-2 films within the spectral wavelengths of $600 \mathrm{~nm}$ to $750 \mathrm{~nm}$ is shown.

Table 1. Dose response of five layers of HS Gafchromic and EDR-2 radiographic film. The net optical density changes for five layers of HS Gafchromic and one layer of EDR-2 radiographic film with two specific bandwidths of $10 \mathrm{~nm}$ and $30 \mathrm{~nm}$ located around $675 \mathrm{~nm}$ are shown.

\begin{tabular}{rllll}
\hline & \multicolumn{4}{c}{ Net optical density } \\
\cline { 2 - 5 } Dose (cGy) & HS $(10 \mathrm{~nm})$ & HS $(30 \mathrm{~nm})$ & EDR-2 $(10 \mathrm{~nm})$ & EDR-2 $(30 \mathrm{~nm})$ \\
\hline 0 & 0 & 0 & 0 & 0 \\
50 & 0.252 & 0.196 & 0.135 & 0.13 \\
100 & 0.511 & 0.384 & 0.294 & 0.289 \\
150 & 0.733 & 0.572 & 0.482 & 0.475 \\
200 & 0.986 & 0.773 & 0.66 & 0.652 \\
250 & 1.23 & 0.971 & 0.84 & 0.829 \\
300 & 1.49 & 1.17 & 1.026 & 1.014 \\
\hline
\end{tabular}

film scanners use LEDs which have a finite bandwidth, the dose response of both films was examined with bandwidths of $10 \mathrm{~nm}$ and $30 \mathrm{~nm}$ around the $675 \mathrm{~nm}$ absorption peak. The results for dose response are quoted in table 1 . The results show that even with a finiteband width, the five layers of HS film still provide a dosimeter with a dose sensitivity which is similar to that of the EDR-2 radiographic film. If the entire visible spectrum is taken into account the sensitivity response to dose for the five layers of HS Gafchromic is calculated as $0.165 \mathrm{OD} / \mathrm{Gy}$ compared to $0.295 \mathrm{OD} / \mathrm{Gy}$ for EDR-2 radiographic film. This is approximately a factor of 2 lower response for HS compared to EDR-2, which would be the case for visible light densitometers. The HS Gafchromic film, due to its relatively low energy dependence (approximately $2 / 3 \mathrm{rd}$ dose response at $100 \mathrm{kVp}$ compared to $6 \mathrm{MV}$ ), has an advantage over EDR-2 film (energy response of approximately $10 \times$ larger at $100 \mathrm{kVp}$ compared to $6 \mathrm{MV}$ ) for measurements in regions where the spectral distribution of the beam is unknown and may contain a higher level of lower energy scattered radiation such as around multileaf collimators used in conformal therapy or IMRT. With the high sensitivity shown, the dose response of the five HS layers is greater than EDR-2 film when read at its wavelength of maximum absorption, which provides good dosimetric characteristics for applications such as IMRT dose verification. 


\section{Conclusion}

Five layers of the relatively new HS Gafchromic film provide a dose sensitivity which can exceed that of EDR-2 radiographic film at a specific chosen wavelength (0.55 OD/Gy) and does not produce the high over response exhibited by conventional radiographic film at low energies. Thus it could be useful for applications such as IMRT dose verification. Doses around devices such as multileaf collimators where the spectral components of the beam are not fully known and could contain a greater low energy component could be more accurately measured with the five layers of HS film.

\section{Acknowledgment}

This work has been fully supported by a grant from the Research Grants Council of HKSAR, China (Project No CityU 1012/01P).

\section{References}

Butson M, Yu P and Metcalfe P 1998 Effects of ambient and readout light sources on Gafchromic film Phys. Med. Biol. 43 2407-12

Butson M, Cheung T and Yu P 2002 High sensitivity radiochromic film dose comparisons Phys. Med. Biol. 47 N291-5

Constantinou C, Attix F H and Paliwal B R 1982 A solid water phantom material for radiotherapy x-ray and gamma ray beam ray calculations $\mathrm{Med}$. Phys. 9 436-41

Meigooni A, Sanders M, Ibbott G and Szeglin S 1996 Dosimetric characteristics of an improved radiochromic film Med. Phys. 23 1883-8

McLaughlin W, Yun -Dong C, Soares C, Miller A, Dyk G and Lewis D 1991 Sensitometry of the response of a new radiochromic film dosimeter to gamma radiation and electron beams Nucl. Instrum. Methods Phys. Res. A 302 165-76

Niroomand-Rad A, Blackwell C, Coursey B, Gall K, Galvin J, McLaughlin W, Meigooni A, Nath R, Rodgers J and Soares C 1998 Radiochromic film dosimetry : recommendation of AAPM radiation therapy task group 55 Med. Phys. 25 2093-115

Reinstein L, Gluckman G, Pai S and Meek A 1997 Post irradiation dependance of radiochromic film optical density on temperature and time Med. Phys. 241005

Zhu Y, Kirov A, Mishra A, Meigooni A and Williamson J 1997 Quantitative evaluation of radiochromic film response for two-dimensional dosmietry Med. Phys. 24 223-31 